\title{
Reflection for Meniere's Disease in the Era of Modern Precision Medicine
}

\author{
Juen-Haur Hwang, MD, PhD1,2,3*
}

${ }^{1}$ Department of Otolaryngology-Head and Neck Surgery, Dalin Tzu Chi Hospital, Chiayi, Taiwan ${ }^{2}$ School of Medicine, Tzu Chi University, Hualien, Taiwan

${ }^{3}$ Department of Medical Research, China Medical University Hospital, China Medical University, Taichung, Taiwan

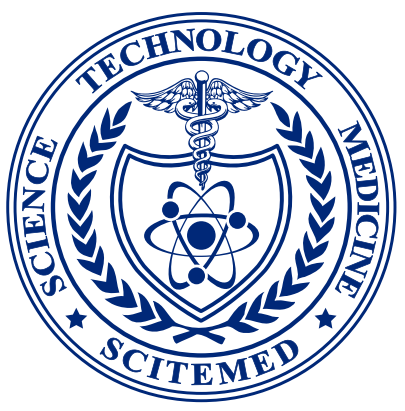

\begin{abstract}
Many diseases have been renamed on the basis of their anatomical sites and pathophysiological findings, as understanding of their underlying mechanisms has improved over time. One of them is Meniere's disease (MD), a well-known vertiginous disease named after the French doctor, Prosper Meniere, who had suggested in 1861 that its symptoms came from the inner ear and not the brain, as was the prevailing belief at that time. This symptom-based diagnosis for MD was against its pathological findings and raised one important issue: whether MD was a specific inner ear disease or was it just a general description for an inner ear disease with deafness and vertigo? The prevailing inclusion criteria for MD were not specific and diagnostic practices tended to restrict MD as a specific inner ear disease by excluding known inner ear and/or brain etiologies. This exclusion criteria were against Dr. Meniere's original concept, who had emphasized the fact of vertigo could be caused by inner ear problem. Thus, it was believed that MD was a general description for an inner ear disease with deafness and vertigo. In the era of modern precision medicine, it is recommended that doctors inform patients with deafness and vertigo about the diseased site and the related pathological changes instead of a diagnosis of MD.
\end{abstract}

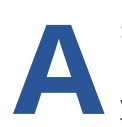
s understanding of the underlying mechanisms of diseases has improved, many diseases have been renamed over the years on the basis of their anatomical sites and/or pathophysiological findings while building on the contributions of previous researchers and/or doctors. Meniere's disease (MD) is a well-known traditional disease that was named after Dr. Prosper Meniere in 1872 [1]. In an article published in 1861, Dr. Meniere had reported a type of deafness with vertigo and suggested that, in addition to brain disorders, vertigo could also originate from inner ear problems. Hence deafness and vertigo that originated from the inner ear still carries Dr. Meniere's name in recognition of his contribution. However, the underlying mechanisms of MD were unclear at that time. In 1938, research papers published by Yamakawa et al. and Hallpike and Cairns found that endolymphatic hydrops might account for MD [1]. As understanding of the disease improved, it was increasingly understood that MD was a kind of deafness and vertigo which could be caused by endolymphatic hydrops in the inner ear. Thereafter, it has been defined by symptomatic criteria since 1972. Further, the symptomatic criteria for the disease have been revised several times in 1985, 1995, and 2015 [2].

The symptom-based diagnosis goes somewhat against pathological findings. It raised an important issue: whether MD was a specific inner ear disease or was it just a general description for an inner ear disease with deafness and vertigo? The prevailing symptomatic inclusion criteria were not very specific, as some other inner ear and/ or brain diseases could also be diagnosed as MD, such as transient ischemic attack (TIA) [2], vestibular migraine (VM), cochleovestibular migraine (CVM) [3], and other similar conditions. Hence, the symptomatic criteria tended to exclude all known inner ear and/or brain etiologies, restricting MD as a unique form of inner ear disease. Unless the etiology and/or pathological changes underlying deafness and vertigo became clear, the diagnostic philosophy continued to define MD as simply a collection of inner ear disease with deafness and vertigo of unknown etiology. Most importantly, the exclusion criteria for the disease were against Dr. Meniere's concept, which had emphasized that vertigo could be caused by inner ear problem. Thus, we believed that MD was just a general description for an inner ear disease with deafness and vertigo. However, many subsequent studies had found that MD was often comorbid in patients with arthritis, psoriasis, migraine, gastroesophageal reflux disease, irritable bowel syndrome, and chronic kidney disease [2]. Thus, MD was also a mixed-type inner ear disease of deafness and vertigo, as was commonly believed, and the exclusion of known etiology during diagnosis was not justified.

The pathophysiology of endolymphatic hydrops and its association with MD remains elusive [4]. The symptoms of most patients with MD become noticeable only after a considerable accumulation of endolymph. However, the diagnosis of MD based on symptomatic inclusion and exclusion criteria does not reflect the existence of endolymphatic hydrops either by electrocochleography (ECOG) [5] or by magnetic resonance imaging (MRI) [6]. It was observed that the ratio of summation potential versus action potential (SP/AP ratio) was not elevated but matched for MD in 43.9\% (29/66) of the MD patients based on symptomatic criteria [5]. The inversion of the saccule to utricle area ratio (SURI) on the axial 3D-FLAIR MRI was found only in 50\% (15/30) patients [6]. On the other hand, asymptomatic endolymphatic hydrops might be found on the unaffected side of patients with unilateral MD $[4,5]$. Hence, patients with "asymptomatic" endolymphatic hydrops are not necessarily the same as those with "symptomatic" MD. Thus, it may be difficult to attribute the etiology of the patients with MD solely to endolymphatic hydrops. Meanwhile, the condition of endolymphatic hydrops is not restricted to patients with typical MD but can also be present in other conditions such as atypical MD, large vestibular aqueduct syndrome, superior canal dehiscence syndrome, low cerebrospinal fluid pressure syndrome and perilymphatic fistula [4].

Based on above discussion, it was believed that MD was just a general description for an inner ear disease with deafness and vertigo. In the era of modern precision medicine, we would encourage doctors to inform their patients with deafness and vertigo about the diseased sites and the related pathological changes instead of a general diagnosis of MD only. 


\section{ARTICLE INFORMATION}

*Correspondence:Juen-Haur Hwang, MD, PhD, Department of Otolaryngology-Head and Neck Surgery, Dalin Tzu Chi Hospital, Buddhist Tzu Chi Medical Foundation, No. 2, Minsheng Road, Dalin, Chiayi, 62247, Taiwan. Email: g120796@tzuchi.com.tw

Received: Nov. 02, 2021; Accepted: Dec. 06, 2021; Published: Dec. 14, 2021

DOI: $10.24983 /$ scitemed.aohns.2021.00150

Ethics Approval and Consent to Participate: The study is in accordance with the ethical standards of the 1964 Helsinki declaration and its later amendments or comparable ethical standards.

Funding: This research received no specific grant from any funding agency in the public, commercial, or not-for-profit sectors.

Conflict of Interest: The author reports no financial or other conflict of interest relevant to this article, which is the intellectual property of the author.

Copyright $@ 2021$ The Author. This is an open-access article distributed under the terms of the Creative Commons Attribution 4.0 International License (CC-BY).

\section{REFERENCES}

1. Lustig LR, Lalwani A. The history of Meniere's disease. Otolaryngol Clin North Am 1997;30(6):917-945.

2. Lopez-Escamez JA, Carey J, Chung WH, et al. Diagnostic criteria for Meniere's disease. J Vestib Res 2015;25(1):1-7.

3. Hwang JH, Tsai SJ, Liu TC, Chen YC, Lai JT. Association of Tinnitus and Other Cochlear Disorders With a History of Migraines. JAMA Otolaryngol Head Neck Surg 2018;144(8):712-717.

4. Liu Y, Yang J, Duan M. Current status on researches of Meniere's disease: A review. Acta Otolaryngol 2020;140(10):808-812.

5. Hwang JH, Lin YC, Lee QC, Hsiao $\mathrm{SH}, \mathrm{Ho} \mathrm{CH}$. [Correlation between extratympanic electrocochleography results and disease side in unilateral Meniere's disease]. J Taiwan Otolaryngol-Head Neck Surg 2009;44(2):46-50.

6. Attye A, Eliezer M, Boudiaf N, et al. MRI of endolymphatic hydrops in patients with Meniere's disease: A case-controlled study with a simplified classification based on saccular morphology. Eur Radiol 2017;27(8):3138-3146. 